PROCEEDINGS OF THE

AMERICAN MATHEMATICAL SOCIETY

Volume 137, Number 7, July 2009, Pages 2419-2424

S 0002-9939(09)09821-9

Article electronically published on January 28, 2009

\title{
MOSER STABILITY FOR LOCALLY CONFORMALLY SYMPLECTIC STRUCTURES
}

\author{
G. BANDE AND D. KOTSCHICK \\ (Communicated by Jon G. Wolfson)
}

\begin{abstract}
We formulate and prove the analogue of Moser's stability theorem for locally conformally symplectic structures. As special cases we recover some results previously proved by Banyaga.
\end{abstract}

\section{INTRODUCTION}

In this paper we prove a version of the Moser stability theorem for locally conformally symplectic structures. These structures were introduced by Lee [6] and Vaisman 8 and have been studied extensively by Vaisman, Banyaga and many others. We refer the reader to [1, 2, 3, 5, 6, 8, and to the references given there for a more thorough discussion.

A locally conformally symplectic or les form on a manifold $M$ is a non-degenerate 2 -form $\omega$ which is locally conformal to a symplectic form. More formally:

Definition 1.1. A non-degenerate 2 -form $\omega$ on a manifold $M$ is said to be locally conformally symplectic if there exists an open covering $\left\{U_{i}\right\}$ of $M$ and a smooth positive function $f_{i}$ on each $U_{i}$ such that $\left.f_{i} \omega\right|_{U_{i}}$ is symplectic on $U_{i}$.

It is straightforward to see, and was first observed by Lee [6], that this is equivalent to the existence of a closed 1-form $\theta$ such that

$$
d \omega=\theta \wedge \omega .
$$

We assume throughout that the dimension of $M$ is at least 4 . Then the 1 -form $\theta$, called the Lee form of $\omega$, is uniquely determined by $\omega$ because the wedge product with a non-degenerate 2 -form is injective on 1 -forms. When $\theta$ vanishes identically, the form $\omega$ is symplectic.

Two lcs forms $\omega$ and $\omega^{\prime}$ are said to be (conformally) equivalent if there exists some positive function $f$ such that $\omega=f \omega^{\prime}$. A locally conformally symplectic structure is an equivalence class of lcs forms for this relation. Note that the de Rham cohomology class of the Lee form is an invariant of the lcs structure because a conformal rescaling of $\omega$ changes $\theta$ by the addition of an exact form.

Received by the editors October 8, 2008 .

2000 Mathematics Subject Classification. Primary 53D99; Secondary 57R17, 58H15.

This work was carried out while the second author was a Visiting Professor at the Università degli Studi di Cagliari, supported by the Regione Autonoma della Sardegna.

(C)2009 G. Bande and D. Kotschick 
If an lcs structure contains a symplectic representative, then the structure is globally conformally symplectic. This is the case if and only if the Lee form is exact.

The purpose of this paper is to give a necessary and sufficient condition for the existence of an isotopy making a smooth family of lcs structures constant. This result, Theorem 3.1 proved in Section 3, is the lcs analogue of Moser's theorem [7] for symplectic forms. However, for the conformally invariant notion of lcs forms, the appropriate formulation is not for an isotopy of forms, but for an isotopy of their conformal equivalence classes. We will show that Theorem 3.1 implies as special cases some results of Banyaga 1 giving sufficient conditions for the existence of an isotopy of certain families of lcs structures. Although we prove a more general result than Banyaga [1, our proofs are simpler, even for the special cases he considered; compare Section 4 below.

The proof of Theorem 3.1 involves Hodge theory for the Lichnerowicz cohomology, which we review in Section 2, As a byproduct we give an answer to a question raised by Banyaga in [2].

To end this introduction, let us remark on the notation we use. There are a number of conventions, especially concerning signs, involved in the definition of the Lee form and in the discussion of Lichnerowicz cohomology. We use (1.1) as our definition of the Lee form, and we have chosen the sign conventions for the Lichnerowicz cohomology in such a way that they fit conveniently with (1.1).

\section{LiCHNEROWICZ COHOMOLOGY}

An important tool in the study of lcs structures is the $d_{\theta}$-cohomology introduced in [4]; compare also [2, 3, 5].

Let $M$ be a smooth manifold, and $\theta$ a closed 1-form on $M$. One defines a first-order differential operator $d_{\theta}$ as follows:

$$
d_{\theta} \beta=d \beta-\theta \wedge \beta,
$$

where $\beta$ is any differential form. It is straightforward to verify that $d_{\theta}$ squares to zero, so that one obtains a modified de Rham complex $\left(\Omega^{*}(M), d_{\theta}\right)$. Its cohomology vector spaces $H_{\theta}^{*}(M)$ are called the $d_{\theta}$-cohomology, or Lichnerowicz cohomology of $M$ with respect to $\theta$. This only depends on the de Rham cohomology class of $\theta$, for if $\theta^{\prime}=\theta+d \ln f$ for some positive function $f$, then the formula

$$
f d_{\theta} \beta=d_{\theta+d \ln f}(f \beta)
$$

shows that multiplication by $f$ is a chain map between $\left(\Omega^{*}(M), d_{\theta}\right)$ and $\left(\Omega^{*}(M), d_{\theta^{\prime}}\right)$ inducing an isomorphism in cohomology.

In the case when $\theta$ is the Lee form of an lcs form $\omega$, equation (1.1) shows that $\omega$ is $d_{\theta}$-closed and so defines a class in $H_{\theta}^{2}(M)$. If we consider the lcs structure defined by $\omega$, and $\omega^{\prime}=f \omega$, then the Lee form of $\omega^{\prime}$ is just $\theta^{\prime}=\theta+d \ln f$ and the class $[\omega] \in H_{\theta}^{2}(M)$ is mapped to $\left[\omega^{\prime}\right] \in H_{\theta^{\prime}}^{2}(M)$ by the above isomorphism.

The Lichnerowicz cohomology shares many properties with the ordinary de Rham cohomology; see, for example, 4, 5. For our purposes it is useful that Hodge theory applies to the $d_{\theta}$-cohomology.

Let us assume that $M$ is closed and oriented. Then the modified de Rham complex $\left(\Omega^{*}(M), d_{\theta}\right)$ is an elliptic complex. In particular, its cohomology is finitedimensional. If we equip $M$ with an arbitrary Riemannian metric $g$, then we can define an operator $d_{\theta}^{*}$ as the formal $L^{2}$-adjoint of $d_{\theta}$ with respect to $g$. Further, 
$\Delta_{\theta}=d_{\theta} d_{\theta}^{*}+d_{\theta}^{*} d_{\theta}$ is the corresponding Laplacian. These operators are lower-order perturbations of the corresponding operators in the usual Hodge-de Rham theory (corresponding to $\theta=0$ ) and therefore have much the same analytic properties. For example, the usual proof of the Hodge decomposition theorem (see e.g. 9]) goes through, and one obtains an orthogonal decomposition

$$
\Omega^{k}(M)=\mathcal{H}^{k}(M) \oplus d_{\theta}\left(\Omega^{k-1}(M)\right) \oplus d_{\theta}^{*}\left(\Omega^{k+1}(M)\right),
$$

where $\mathcal{H}^{k}(M)$, the space of $\Delta_{\theta}$-harmonic forms, is isomorphic to $H_{\theta}^{k}(M)$.

Any $d_{\theta}$-exact $k$-form is contained in $d_{\theta}\left(\Omega^{k-1}(M)\right)$, which is in fact equal to $d_{\theta} d_{\theta}^{*}\left(\Omega^{k}(M)\right)$. Integration by parts shows that

$$
d_{\theta}: d_{\theta}^{*}\left(\Omega^{k}(M)\right) \longrightarrow \Omega^{k}(M)
$$

is injective, so that every $d_{\theta}$-exact form has a unique primitive in the image of $d_{\theta}^{*}$.

Finally, note that on a closed oriented manifold the index of the elliptic complex $\left(\Omega^{*}(M), d_{\theta}\right)$ is determined, via the Atiyah-Singer index theorem, by its symbol sequence, which is independent of $\theta$. Therefore, the Euler characteristic of the Lichnerowicz cohomology coincides with the usual Euler characteristic.

Example 2.1. Banyaga 2] considers the Lichnerowicz cohomology on a certain 4-manifold $M$ of Euler characteristic zero. For a particular closed one-form $\theta$ he shows that the dimensions of $H_{\theta}^{i}(M)$ are at least one for $i=1,2,3$, and asks whether the dimensions might be exactly one; see Question 3 on page 5 of [2].

In this case the one-form $\theta$ is not exact, and so a result of [4, 5] shows that $H_{\theta}^{i}(M)$ vanishes for $i=0$ and $i=4$. Therefore the vanishing of the Euler characteristic implies that $H_{\theta}^{2}(M)$ is at least 2-dimensional, giving a negative answer to Banyaga's question.

\section{Moser Stability}

In this section we consider families $\omega_{t}$ of locally conformally symplectic forms depending smoothly on a parameter $t \in[0,1]$. The uniqueness of the Lee form $\theta_{t}$ implies that this depends smoothly on $t$ as well.

Recall that Moser's stability theorem [7] says that if the $\omega_{t}$ are actually symplectic, then they are isotopic as forms if and only if their de Rham cohomology class is independent of $t$. Now one expects to transpose this statement to the lcs category by replacing the de Rham cohomology by the Lichnerowicz cohomology. This is slightly complicated for two reasons. First of all, the Lichnerowicz cohomology depends on $\theta_{t}$, which is not necessarily fixed, but varies with $t$. Thus the cohomology one needs to consider also varies with $t$. Second of all, we would like to have a conformally invariant statement, which holds for lcs structures rather than forms. This explains why the following theorem looks more complicated than Moser's.

Theorem 3.1. Let $\omega_{t}$ be a family of locally conformally symplectic forms on a closed manifold $M$, depending smoothly on $t \in[0,1]$. Denote by $\theta_{t}$ the Lee form of $\omega_{t}$.

There exists an isotopy $\phi_{t}$ with $\phi_{t}^{*} \omega_{t}$ conformally equivalent to $\omega_{0}$ for all $t$ if and only if there are positive smooth functions $f_{t}$ on $M$, varying smoothly with $t$, such that the time derivative $\frac{d}{d t}\left(f_{t} \omega_{t}\right)$ of the conformally rescaled family $f_{t} \omega_{t}$ is $d_{\theta_{t}^{\prime}}$-exact for every $t$, where $\theta_{t}^{\prime}=\theta_{t}+d \ln f_{t}$ is the Lee form of $f_{t} \omega_{t}$. 
Proof. First suppose that there is an isotopy $\phi_{t}$ so that $\phi_{t}^{*} \omega_{t}$ is conformally equivalent to $\omega_{0}$ for all $t$. After rescaling the $\omega_{t}$ suitably, we may assume $\phi_{t}^{*} \omega_{t}=\omega_{0}$. Let $X_{t}$ be the time-dependent vector field obtained by differentiating $\phi_{t}$. By the Cartan formula we have

$$
\begin{aligned}
0 & =\frac{d}{d t}\left(\phi_{t}^{*} \omega_{t}\right)=\phi_{t}^{*}\left(\dot{\omega}_{t}+L_{X_{t}} \omega_{t}\right) \\
& =\phi_{t}^{*}\left(\dot{\omega}_{t}+d i_{X_{t}} \omega_{t}+i_{X_{t}} d \omega_{t}\right) \\
& =\phi_{t}^{*}\left(\dot{\omega}_{t}+d i_{X_{t}} \omega_{t}+i_{X_{t}}\left(\omega_{t} \wedge \theta_{t}\right)\right) \\
& =\phi_{t}^{*}\left(\dot{\omega}_{t}+d i_{X_{t}} \omega_{t}-\theta_{t} \wedge\left(i_{X_{t}} \omega_{t}\right)+\theta_{t}\left(X_{t}\right) \omega_{t}\right) .
\end{aligned}
$$

We conclude that

$$
\dot{\omega}_{t}=-d_{\theta_{t}}\left(i_{X_{t}} \omega_{t}\right)-\theta_{t}\left(X_{t}\right) \omega_{t}
$$

Now consider the family $f_{t} \omega_{t}$ with

$$
f_{t}=\exp \left(\int_{0}^{t} \theta_{s}\left(X_{s}\right) d s\right) .
$$

This satisfies

$$
\begin{aligned}
\frac{d}{d t}\left(f_{t} \omega_{t}\right) & =\dot{f}_{t} \omega_{t}+f_{t} \dot{\omega}_{t} \\
& =f_{t} \theta_{t}\left(X_{t}\right) \omega_{t}-f_{t}\left(d_{\theta_{t}}\left(i_{X_{t}} \omega_{t}\right)+\theta_{t}\left(X_{t}\right) \omega_{t}\right) \\
& =-f_{t} d_{\theta_{t}}\left(i_{X_{t}} \omega_{t}\right) \\
& =-d_{\theta_{t}+d \ln f_{t}}\left(f_{t} i_{X_{t}} \omega_{t}\right),
\end{aligned}
$$

where we first used (3.1) and then (2.2). Thus $\frac{d}{d t}\left(f_{t} \omega_{t}\right)$ is indeed $d_{\theta_{t}^{\prime}}$-exact, where $\theta_{t}^{\prime}=\theta_{t}+d \ln f_{t}$ is the Lee form of $f_{t} \omega_{t}$.

For the converse assume that we have rescaled $\omega_{t}$ in such a way that $\dot{\omega}_{t}$ is $d_{\theta_{t}}$ exact for every $t$. As explained in Section 2, by Hodge theory for the Lichnerowicz cohomology, there is a unique $\alpha_{t}$ in the image of $d_{\theta_{t}}^{*}$ with the property that $d_{\theta_{t}} \alpha_{t}=$ $\dot{\omega}_{t}$. As $\dot{\omega}_{t}$ depends smoothly on $t$, and $\alpha_{t}$ is specified uniquely for every $t$, it follows that $\alpha_{t}$ also depends smoothly on $t$. We define a time-dependent vector field $X_{t}$ by the requirement that

$$
i_{X_{t}} \omega_{t}=-\alpha_{t} .
$$

This exists and is unique because $\omega_{t}$ is non-degenerate. As both $\omega_{t}$ and $\alpha_{t}$ depend smoothly on $t$, so does $X_{t}$. Let $\phi_{t}$ be its flow. Now the same calculation as before yields

$$
\begin{aligned}
\frac{d}{d t}\left(\phi_{t}^{*} \omega_{t}\right) & =\phi_{t}^{*}\left(\dot{\omega}_{t}+L_{X_{t}} \omega_{t}\right) \\
& =\phi_{t}^{*}\left(\dot{\omega}_{t}+d i_{X_{t}} \omega_{t}-\theta_{t} \wedge\left(i_{X_{t}} \omega_{t}\right)+\theta_{t}\left(X_{t}\right) \omega_{t}\right) \\
& =\phi_{t}^{*}\left(\dot{\omega}_{t}-d_{\theta_{t}} \alpha_{t}+\theta_{t}\left(X_{t}\right) \omega_{t}\right) \\
& =\phi_{t}^{*}\left(\theta_{t}\left(X_{t}\right)\right) \cdot \phi_{t}^{*} \omega_{t} .
\end{aligned}
$$

It follows that

$$
\phi_{t}^{*} \omega_{t}=\exp \left(\int_{0}^{t} \phi_{s}^{*}\left(\theta_{s}\left(X_{s}\right)\right) d s\right) \cdot \omega_{0} .
$$

This completes the proof. 
Note that in the second part of the proof we produced an isotopy $\phi_{t}$ between the conformal equivalence classes of the $\omega_{t}$. As the de Rham cohomology class of the Lee form is conformally invariant, and $\phi_{t}$ acts trivially on de Rham cohomology, we conclude that the de Rham cohomology class of the Lee forms $\theta_{t}$ is independent of $t$. We do not need to assume this in the theorem, as the proof shows that it is implied by the assumption that $\frac{d}{d t}\left(f_{t} \omega_{t}\right)$ is $d_{\theta_{t}^{\prime}}$-exact for all $t$.

If we assume that the Lee forms are independent of $t$, then Theorem 3.1 implies the following statement, first proved by Banyaga (Theorem 4 of [1]):

Corollary 3.2. Let $\omega_{t}$ be a smooth family of lcs forms on a compact manifold $M$ having the same Lee form $\theta$. If $\omega_{t}-\omega_{0}$ is $d_{\theta}$-exact for all $t$, then there exist a family of functions $f_{t}$ and an isotopy $\phi_{t}$ such that $\phi_{t}^{*}\left(\omega_{t}\right)=f_{t} \omega_{0}$.

Another special case of Theorem 3.1 previously proved by Banyaga is the following (Theorem 5 of [1]):

Corollary 3.3. Let $\omega_{t}$ be a smooth family of lcs forms on a compact manifold $M$ such that the corresponding Lee forms $\theta_{t}$ have the same de Rham cohomology class. Suppose there exists a smooth family of 1-forms $\alpha_{t}$ such that $\omega_{t}=d \alpha_{t}-\theta_{t} \wedge \alpha_{t}$. Then there exists an isotopy $\phi_{t}$ such that $\phi_{t}^{*} \omega_{t}$ is conformally equivalent to $\omega_{0}$ for all $t$.

Proof. In this case we have

$$
\dot{\omega}_{t}=d \dot{\alpha}_{t}-\theta_{t} \wedge \dot{\alpha}_{t}-\dot{\theta}_{t} \wedge \alpha_{t}=d_{\theta_{t}} \dot{\alpha}_{t}-\dot{\theta}_{t} \wedge \alpha_{t} .
$$

Because of the second summand on the right-hand side, it is not obvious that Theorem 3.1 applies. However, here one has the additional assumption that the de Rham cohomology class of $\theta_{t}$ is independent of $t$. This means that $\dot{\theta}_{t}=d h_{t}$ for some smoothly varying family of functions $h_{t}$. Let

$$
g_{t}=-\int_{0}^{t} h_{s} d s
$$

and $f_{t}=\exp g_{t}$. With these definitions we have

$$
\begin{aligned}
\frac{d}{d t}\left(f_{t} \omega_{t}\right) & =\dot{f}_{t} \omega_{t}+f_{t} \dot{\omega}_{t} \\
& =f_{t} \dot{g}_{t} \omega_{t}+f_{t}\left(d_{\theta_{t}} \dot{\alpha}_{t}-\dot{\theta}_{t} \wedge \alpha_{t}\right) \\
& =f_{t}\left(-h_{t}\left(d \alpha_{t}-\theta_{t} \wedge \alpha_{t}\right)+d_{\theta_{t}} \dot{\alpha}_{t}-\dot{\theta}_{t} \wedge \alpha_{t}\right) \\
& =f_{t} d_{\theta_{t}}\left(-h_{t} \alpha_{t}+\dot{\alpha}_{t}\right) \\
& =d_{\theta_{t}+d \ln f_{t}}\left(f_{t}\left(-h_{t} \alpha_{t}+\dot{\alpha}_{t}\right)\right),
\end{aligned}
$$

where we used first (3.2), then $\dot{\theta}_{t}=d h_{t}$, and finally (2.2).

We are now in a position to appeal to the sufficiency proof for the existence of the isotopy from (the proof of) Theorem 3.1. However, as we have an explicit smooth family of $d_{\theta_{t}+d} \ln f_{t}$-primitives for $\frac{d}{d t}\left(f_{t} \omega_{t}\right)$, Hodge theory is not needed.

Remark 3.4. Instead of deducing Corollary 3.3 from Theorem 3.1, one can give a quick direct proof as follows. Given a smooth family of functions $h_{t}$ such that $\dot{\theta}_{t}=d h_{t}$, one defines a time-dependent vector field $X_{t}$ by

$$
i_{X_{t}} \omega_{t}=-\dot{\alpha}_{t}+h_{t} \alpha_{t}
$$


Then its flow $\phi_{t}$ satisfies

$$
\frac{d}{d t}\left(\phi_{t}^{*} \omega_{t}\right)=\phi_{t}^{*}\left(\left(\theta_{t}\left(X_{t}\right)+h_{t}\right) \omega_{t}\right)
$$

by the same kind of calculation as above, and so provides the desired isotopy.

\section{Conclusion}

In this paper we have given a necessary and sufficient condition for the existence of an isotopy $\phi_{t}$ making a smooth family of lcs structures constant. The necessity had not appeared in the literature before. Banyaga [1] had discussed special cases of the sufficiency. Comparing our arguments with his, the main difference is that we work directly on a closed manifold $M$ and construct the desired isotopy by integrating a time-dependent vector field on $M$, whereas Banyaga [1] worked on a covering of $M$ which is no longer compact. The non-compactness leads to two complications. One is that completeness of (time-dependent) vector fields is not always available and care has to be taken to ensure it in the situation at hand. The other complication is that Hodge theory is not available and the only way to find smooth families of primitives for $d_{\theta}$-exact forms is Grothendieck's theory of nuclear spaces.

\section{REFERENCES}

1. A. Banyaga, Some properties of locally conformal symplectic structures, Comment. Math. Helv. 77 (2002), no. 2, 383-398. MR.1915047 (2003d:53134)

2. A. Banyaga, Examples of non $d_{\omega}$-exact locally conformal symplectic forms, J. Geom. $\mathbf{8 7}$ (2007), 1-13. MR2372512(2008i:53113)

3. S. Dragomir and L. Ornea, Locally conformal Kähler geometry, Progress in Mathematics 155, Birkhäuser Boston, Inc., Boston, MA, 1998. MR.1481969 (99a:53081)

4. F. Guédira and A. Lichnerowicz, Géométrie des algèbres de Lie locales de Kirillov, J. Math. Pures Appl. 63 (1984), no. 4, 407-484. MR789560 (86j:58045)

5. S. Haller and T. Rybicki, On the group of diffeomorphisms preserving a locally conformal symplectic structure, Ann. Global Anal. Geom. 17 (1999), no. 5, 475-502. MR 1715157 (2001f:53164)

6. H. C. Lee, A kind of even-dimensional differential geometry and its application to exterior calculus, Amer. J. of Math. 65 (1943), 433-438. MR0008495(5:15h)

7. J. Moser, On the volume elements on a manifold, Trans. Amer. Math. Soc. 120 (1965), 286-294. MR0182927 (32:409)

8. I. Vaisman, Locally conformal symplectic manifolds, Internat. J. Math. Math. Sci. 8 (1985), no. 3, 521-536. MR809073 (87d:53076)

9. F. W. Warner, Foundations of differentiable manifolds and Lie groups, Scott, Foresman and Co., Glenview, IL, and London, 1971. MR0295244 (45:4312)

Dipartimento di Matematica e Informatica, Università degli Studi di Cagliari, Via Ospedale 72, 09124 Cagliari, Italy

E-mail address: gbande@unica.it

Mathematisches Institut, Ludwig-Maximilians-Universität München, TheresienSTR. 39, 80333 MÜnChen, Germany

E-mail address: dieter@member.ams.org 